nine of these had their swabs taken eight or more days after onset of illness. Excluding samples taken after eight days, the sensitivity of PCR compared with any positive diagnosis (PCR, serology, and culture) was 94.2\%. Conversely, 12 patients had positive results with PCR, but had negative results with serology. Thus, serology sensitivity compared with any positive result was $88.7 \%$.

An additional $10 \%$ of samples were positive by PCR in lysis buffer alone.

Five of 13 samples that were negative for influenza and that were submitted in the first two weeks of the study were later confirmed by PCR to be rhinovirus infections.

\section{Comment}

Real time surveillance using PCR with a rapid turnaround time confirms that influenza is circulating. As the PCR results were faxed back the next day, there was a stimulus to send in further samples. Results of serology and PCR correlated well, although serology took three weeks longer (figure). No false positives were generated by PCR. Culture was insensitive and slow because of the variation between batches of the primary cell line used in this laboratory. The time since onset of illness is critical for the sensitivity of virus isolation and PCR. In the late phase of illness, when results of culture and PCR were negative, there was already a high antibody titre.

Although culture is required to accumulate virus isolates for antigenic characterisation of the circulating viruses, PCR should now be the front line assay for diagnosis of influenza, even in non-specialist laboratories after initial training. It is clear that additional pathogens cause influenza-like illness, and the introduction of a multiplex PCR to test for a wider number of pathogens ${ }^{24}$ will considerably improve surveillance of the winter respiratory burden. New treatments for influenza strengthen the case for improved virological surveillance to alert clinicians to the cause of influenza-like illness and for rapid diagnosis and appropriate treatment of individual cases.

We acknowledge the hard work performed by the staff of the participating general practices.

Contributors: WFC, LAW, JDD, JM, AN, and PC designed and carried out the study. LAW and JW did the laboratory work. SM coordinated sample collection in all practices. LAW and WFC wrote the paper. WFC guarantees the paper.

Funding: The Scottish Centre for Infection and Environmental Health provided partial financial support.

Competing interests: None declared.

1 Atmar RL, Baxter BD, Dominguez EA, Taber LH. Comparison of reverse transcription-PCR with tissue culture and other rapid diagnostic assays for detection of type A influenza virus. J Clin Microbiol 1996;34:2604-6.

2 Stockton J, Ellis JS, Saville M, Clewley JP, Zambon MC. Multiplex PCR for typing and subtyping influenza and respiratory syncytial viruses. $J$ Clin Microbiol 1998:36:2990-5.

3 Wallace LA, McAulay KA, Douglas JDM, Elder AG, Stott DJ, Carman WF. Influenza diagnosis: from dark isolation into the molecular light. J Infect 1999;39:221-6.

4 Grondahl B, Puppe W, Hoppe A, Kuhne I, Weigl JA, Schmitt HJ. Rapid identification of nine microorganisms causing acute respiratory tract infections by single-tube multiplex reverse transcription-PCR: feasibility study. J Clin Microbiol 1999;37:1-7.

(Accepted 28 March 2000)

\title{
Clinical governance in primary care Participating in clinical governance
}

\author{
Mike Pringle
}

Earlier papers in this series have highlighted the collective nature of clinical governance in primary care and the central role of primary care groups, trusts, and practices. But definitions of clinical governance have also emphasised the responsibilities of individuals, highlighting the importance of lifelong learning by all clinicians and creating a clear obligation actively to manage poor professional performance when it is identified. ${ }^{1-3}$

This article will concentrate on the implications of clinical governance for individuals working in primary care. All primary care practitioners will have to develop systems for clinical governance. This includes pharmacists, opticians, and dentists, for whom the relative isolation of their practices can make such work difficult. ${ }^{4}$ The main focus of this paper will be on clinical governance in general practice.

The paper will explore the relation between clinical governance, continuing professional development, and revalidation. It will consider how an individual's learning and development can be linked to that of the practice as a whole. It will also consider the need to protect the public from unacceptable care and the need to manage poor performance.

\section{Summary points}

Clinical governance is intended to improve standards of care and at the same time to protect the public from unacceptable care

The move from continuing medical education for doctors to continuing professional development for the whole primary care team presents new challenges for multidisciplinary learning and performance monitoring

To deal with poor performance, clinical governance leaders will need skills to assess the nature of the problem, educational resources to deal with it, and managerial resources to facilitate the process

Participation in the activities of clinical governance will be an essential feature of revalidation
This is the

fourth in a series of five articles

Department of General Practice, Queen's Medical Centre, Nottingham NH7 2UH

Mike Pringle professor mike.pringle@ nottingham.ac.uk Series editor: Rebecca Rosen

BMJ 2000;321:737-40 


\section{Developing individual clinicians in primary care}

Clinical governance represents a new formulation of age-old activities. The Royal College of Nursing established a range of quality improvement systems in the 1980s, including the practitioner led dynamic standard setting system ${ }^{5}$ and mechanisms for clinical supervision and reflective practice. ${ }^{6}$ The Royal College of General Practitioners has developed assessment mechanisms for general practitioners linked to objective national standards. ${ }^{78}$ The College of Optometrists and Royal Pharmaceutical Society have established programmes of continuing education and standards for professional practice for opticians and pharmacists respectively. Furthermore, a 1998 report from the chief medical officer signalled a transition from continuing medical education for doctors to continuous professional development for all clinicians. ${ }^{9}$

Clinical governance aims to integrate these various systems for quality improvement and professional development and to ensure that everyone in the practice team becomes involved. Indeed, an underlying challenge for clinical governance in primary care is to move away from professional development based on unidisciplinary education towards multidisciplinary, team based learning. However, Gillam et al highlight potential problems with multidisciplinary learning in general practice. ${ }^{10}$ These include issues of hierarchy, gender, and varied educational achievements in team members, all of which may act as barriers to effective learning.

\section{Team based learning}

Until recently, continuing medical education in general practice rewarded general practitioners for attending educational events and neglected the needs of practice nurses and other team members. The new emphasis on continuing professional development for all clinicians-be they doctors, dentists, nurses, or professionals allied to medicine-will encourage clinicians to reflect on their educational needs and meet those needs. As the box shows, this is an active, not a passive, process where reflection on care leads to the identification of weaknesses that are recorded as educational needs. These can be met in a variety of ways, including through traditional lectures, but may be best met through peer discussion, referring to published literature, or consulting guidelines.

Significant event monitoring (see box) is a method for identifying educational needs, meeting some of those needs, agreeing improvements to care, and showing that improvements have occurred. Though it requires little time, it does require a degree of trust and mutual support among the members of the practice team. ${ }^{11}$ It is quintessentially a multidisciplinary, team based activity that can bring greater coherence and quality to the patient's experience of primary care.

\section{Mechanisms for professional development}

Practices and individuals can use various systems to demonstrate the quality of their care. Membership by assessment of performance ${ }^{7}$ and fellowship by assessment ${ }^{8}$-both of the Royal College of General Practitioners-allow a general practitioner to be assessed against objective national criteria. The quality practice award ${ }^{12}$ and the forthcoming quality team development award offer the same possibility for practice teams.

Clinical governance leaders at practice and primary care group level will need to be sure that each clinician is taking part in effective continuing professional development and that issues raised in performance monitoring are dealt with. The latter will require comparisons between doctors, between nurses, between practices, and between areas.

One way in which continuing professional development can be facilitated is through an annual appraisal. This should be a formative, professionally led review of strengths and weaknesses rather than a punitive managerial process. Such an appraisal could offer a way to ensure regularly that clinical governance works for all doctors and nurses.

\section{Learning from experience}

We must learn from our experience with clinical audit and the evidence based health care movement. Firstly, these ideas are not new, and we must be careful not to sell them as such. Most primary care practitioners reflect on and improve their care and are therefore already taking part in continuing professional development. Once doctors and nurses are able to see that these changes codify and build on what is already happening there will be less antipathy.

\section{The difference between continuing medical education and continuing professional development}

Scenario 1:

Dr Smith sees that there is a Friday lunchtime session on cardiology at her postgraduate centre. She attends and listens to a talk on the management of heart failure by a local cardiologist. She submits a PGEA form.

Scenario 2:

$\mathrm{Mr}$ Jones comes to see Dr Smith after discharge from hospital after a myocardial infarction. He is taking a drug that Dr Smith doesn't recognise, but a check in $B N F$ shows that it is a new anti-arrhythmic drug. After the consultation she notes this drug in her personal development plan (PDP) portfolio.

She presents Mr Jones' case at a significant event audit meeting. The practice team looks at all aspects of prevention, the coronary itself, and rehabilitation. Everything seems to have been done well, and everyone involved is congratulated. Dr Smith then asks about this new drug. In the discussion, she says that she is not very clear about anti-arrhythmic drugs, and she undertakes to explore this area.

She reads the $B N F$ carefully and looks out a recent review in Drug and Therapeutics Bulletin. She reviews recent articles through Medline and finds a helpful systematic review. She telephones a local cardiologist, who is happy to give her 10 minutes to clarify a few issues. She writes a short summary for her practice. This process took less time than a Friday lunchtime lecture. Dr Smith records it in her PDP.

At the next significant event audit meeting the practice team discusses her short report and agrees a protocol. A receptionist is asked to audit the use of anti-arrhythmic drugs on the practice's computer. As a result of this audit, the notes of several patients are marked for review at their next consultation. Several team members record their part in this learning process in their PDPs. 


\section{The team based approach to quality}

Every clinical member of the team was disturbed and concerned by the termination of a pregnancy in a 13 -year-old girl registered with the practice. At a significant event meeting the girl's notes were examined and two possible opportunities to discuss contraception were found-but there was no record of such advice being given. A practice nurse reported that, when seen after her termination, the girl knew about emergency contraception but not how to get it.

The practice asked a doctor, a nurse, the health visitor, and the midwife to look at teenage sexual health. They looked at the literature and available guidelines. They carried out a questionnaire survey in two local schools and they organised an open evening meeting on the topic. They proposed three key areas for change:

- All doctors and nurses should record data such as smoking, alcohol use, and contraception in all teenagers (from 13 onwards) as they do for older patients.

- Posters that promote awareness of contraception services and emergency contraception were put in the waiting room and offered to the local schools and the youth club.

- An early evening teenage health clinic was started by a practice nurse and the health visitor to promote healthy lifestyles, safe sex, contraception, immunisations, and cervical cytology.

The practice now audits the recording on the computer of data about the lifestyles and risk factors for teenagers and attendance at the teenage health clinic. The team believes that it has greatly enhanced the services it offers to this group.

Secondly, we must recognise that these changes will need a great deal of support. This will include training and access to the necessary tools; incentives to participate; protected time (that will involve more than just the transfer of postgraduate educational allowance to this activity); and rewards for success. Without such investment the uptake will be patchy and momentum will be low.

One way that these innovations can be realistically delivered, given the improbability of substantial extra resources, is to exploit those facilities already available. Practice teams seldom fully use the skills and enthusiasm of team members for quality improvement. We have resources in health authorities and primary care groups, and we have strong educational structures. The academic departments of general practice and nursing will have access to evidence and guidelines, and many organisations involve their members in small group work. We will need to take advantage of all available skills and opportunities if these transitions are to be effective.

\section{Protecting the public from unacceptable care}

Most good practices and practice team members should see clinical governance as a positive reinforcement of their drive for improved quality. However there will be times when poor care is revealed and this must be addressed promptly and effectively. If performance is sufficiently bad, action to protect patients must be taken.
The precise system for regulating underperforming doctors in Britain is in a state of flux. The General Medical Council has moved from responding to disasters when they have occurred to dealing with poor performance, hopefully before it has seriously damaged patients. One element of this is revalidation. ${ }^{13}$ However, the chief medical officer has put forward another model in which the NHS fulfils the lead role in addressing poor performance. ${ }^{14}$

The Royal College of General Practitioners and the General Practitioners Committee have proposed a partnership model for the regulation of doctors in which the medical profession plays a key role. This has been set out in The Future of Professionally Led Regulation. ${ }^{15}$ Although the regulation of nurses is in transition, managing poor clinical performance has been identified as an important part of clinical governance. ${ }^{16}$ It would be useful if the principles and much of the practice for regulation was common among all health service professions.

Identifying and improving poor performance

Problems with underperformance might become evident through the review of care for continuing professional development, performance monitoring, annual appraisal, patient surveys, complaints, or revalidation. However underperformance is identified, the individual clinician, the practice, and the leader for clinical governance will want to act to ensure that care is improved.

Action will include an assessment of the doctors or nurses in the context in which they work. Some underperformance is due to local deprivation and health inequities, some is due to poor systems of care, and some is due to underresourcing. Where it is due to an individual, the cause may be a health problem or problems with competency or behaviour.

Whichever it is, there needs to be an accurate and agreed "diagnosis" and a "management plan." The latter must, in time, include the capacity to remove a doctor or nurse from active practice, if appropriate, while they are retrained. A locum will be needed to cover patients while a failing clinician is rescued.

The box illustrates an early case of poor practice identified and dealt with through mechanisms

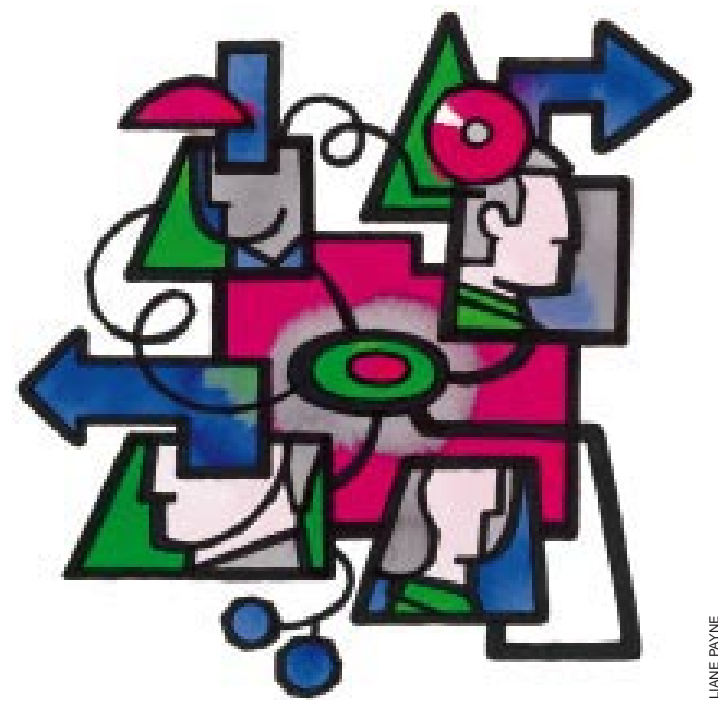




\section{Preventing poor performance}

Dr White is the leader for clinical governance in his primary care group. The prescribing of benzodiazepines in one surgery in the group seemed to be very high so, accompanied by a pharmacist adviser, he visited the practice team. It became clear that Dr Brown, who had a background in psychiatry before entering general practice, dealt with a large number of patients with substance misuse. The discrepancy seemed to be clarified.

However, in discussion Dr Brown explained that she felt stressed and vulnerable; she was unsupported by colleagues and she often felt threatened by patients. She was prescribing inappropriate quantities of benzodiazepines and methadone over sustained periods of time.

With support from the practice and the primary care group, these patients were transferred to other doctors. Dr Brown entered into a period of counselling and returned to full, active practice. A case that might, in a few years' time, have had to be dealt with by the General Medical Council's performance procedures was nipped in the bud.

developed as part of clinical governance. It shows the transition from regulation by exception-waiting for something to go badly wrong before dealing with it-to the new atmosphere of proactive, preventive regulation.

If a problem is severe or the clinician does not respond to offers of help, then the full professional mechanisms will need to be invoked. For general practitioners this will mean referral to the General Medical Council either at the time of revalidation or, if necessary, between revalidation. For nurses similar mechanisms will replace the regulatory role of the United Kingdom Central Committee for Nursing and Health Visiting.

Revalidation, which will be introduced in 2001 for doctors, will offer a regular confirmation of a doctor's fitness to practise. ${ }^{11}$ It will be informed by evidence from continuing professional development, performance monitoring, and other clinical governance work, and it will offer the clinical governance system the ultimate sanction. If a local revalidation group cannot support a doctor's application to continue on the General Medical Council's register then registration may, ultimately, lapse.

Such interventions will be time consuming and expensive. If a general practitioner is taken out of his or her practice and placed in a specially selected and trained practice for supervision, education, and retraining, this will require considerable resources. Practices will also need access to cover for patient care while performance issues are dealt with.

\section{Developing those who will deliver clinical governance}

The tasks of clinical governance are complex and demanding. Those who offer a leadership role will need to be trained and supported. Many will draw on their own experience as clinicians in primary care. Some will attend leadership courses such as those run by the British Association of Medical Managers or the
King's Fund or proposed by the Royal College of General Practitioners.

If we are not to demoralise and lose a generation of primary care leaders, the current generation of those involved in clinical governance needs to be better supported. They need time to do their tasks and they need access to a range of assessment and educational skills. Often general practitioner tutors, directors of postgraduate education for general practitioners, and others possess these skills. However, there is a need for new ways to offer high level support, for example through a local assessment and support service.

\section{Conclusions}

This article has concentrated on clinical governance for general practitioners and general practice, rather than primary care as a whole. That is because general practitioners are the clinicians for whom clinical governance and revalidation will bite earliest.

However, clinical governance will have to become a system that involves everyone in primary care if it is to be effective. At present our understanding of how to promote multidisciplinary learning and education is limited. This represents an important research question which will need to be answered if clinical governance is to develop effectively in primary care. Clinical governance requires the cultural change and increased accountability that have been described earlier in this series. It also requires an increased commitment to the development of people and services, and a renewal of the emphasis on patient care-and the protection of patients-that is at the heart of the health service.

To achieve this change we need professional support, a cadre of leaders with the skills and understanding to deliver clinical governance, and greatly increased resources to apply to underperformance.

1 Secretary of State for Health. The new NHS: modern and dependable. London: Department of Health, 1997.

2 Department of Health. A first class service-quality in the new NHS. London: Department of Health, 1998.

3 Roval College of General Practitioners. Practice advice on the implementation of clinical governance in England and Wales. London: RCGP, 1999.

4 Scrivens E. A scoping study for clinical governance in primary care. Keele: Keele University, 1998.

5 Morrell C, Harvey G, Kitson A. Practitioner based quality improvement: a review of the RCN's dynamic standard setting system. Quality in Health Care 1997;6:9-34.

6 Royal College of Nursing. Guidance for nurses on clinical governance. London: RCN, 1998.

7 Royal College of General Practitioners. Criteria for membership by assessment of performance. London: RCGP, 1999.

8 Royal College of General Practitioners. Criteria for fellowship by assessment. London: RCGP, 1999.

9 Chief Medical Officer. A review of continuing professional development in general practice. London: Department of Health, 1998. www.doh.gov.uk/cmo/ cmodevhtm (accessed 21 July 2000).

10 Gillam S, Eversley J, Snell J, Wallace P. Building bridges. The future of GP education-developing partnerships with the service. London: King's Fund, 1999.

11 Pringle M, Bradley C, Carmichael C, Wallis H, Moore A. Significant event auditing. London: RCGP, 1995. (Occasional paper 70.)

12 Royal College of General Practitioners. Quality practice award. Edinburgh: RCGP, 1999.

13 Royal College of General Practitioners and the General Practitioners Committee. Revalidation for clinical general practice-a consultation document. London: RCGP, 2000.

14 Chief Medical Officer. Supporting doctors, protecting patients. Leeds: Department of Health, 1999

15 Royal College of General Practitioners. The future of professionally led regulation. London: RCGP, 2000.

16 Royal College of Nursing. Guidance for nurses on clinical governance. London: RCN, 1998 